\title{
Optimal control of epidemics with limited resources
}

\author{
Elsa Hansen • Troy Day
}

Received: 8 April 2009 / Revised: 13 March 2010 / Published online: 21 April 2010

(C) The Author(s) 2010. This article is published with open access at Springerlink.com

\begin{abstract}
We extend the existing work on the time-optimal control of the basic SIR epidemic model with mass action contact rate. Previous results have focused on minimizing an objective function that is a linear combination of the cost associated with using control and either the outbreak size or the infectious burden. We instead, provide analytic solutions for the control that minimizes the outbreak size (or infectious burden) under the assumption that there are limited control resources. We provide optimal control policies for an isolation only model, a vaccination only model and a combined isolation-vaccination model (or mixed model). The optimal policies described here contain many interesting features especially when compared to previous analyses. For example, under certain circumstances the optimal isolation only policy is not unique. Furthermore the optimal mixed policy is not simply a combination of the optimal isolation only policy and the optimal vaccination only policy. The results presented here also highlight a number of areas that warrant further study and emphasize that time-optimal control of the basic SIR model is still not fully understood.
\end{abstract}

Keywords Time-optimal control · SIR model · Limited resources · Vaccination · Isolation

Mathematics Subject Classification (2000) $\quad 49 \cdot 92$

\section{Introduction}

Mathematical analyses can provide valuable information about how best to control infectious disease outbreaks. One issue of very real practical concern, and for which

E. Hansen $(\bowtie) \cdot$ T. Day

Department of Mathematics and Statistics, Queen's University,

Jeffery Hall, Kingston, ON K7L 3N6, Canada

e-mail: elsa@mast.queensu.ca 
mathematical modeling has much to offer, is in determining the optimal distribution of limited resources during an outbreak. Commonly, in preparation for an outbreak, a fixed amount of vaccine and other drugs are stockpiled, in addition to the allocation of a certain amount of funds for other control measures such as isolation and quarantine. Once the epidemic starts, the goal is then to optimally administer these resources given that their supply is limited.

One common aim when administering limited resources is to identify a sub-population within the general population that is best targeted to receive the control resources. Consider for example the Polio outbreak in the Netherlands in 1993-1994 (Oostvoigel et al. 1994). This outbreak was concentrated in an unvaccinated sub-population, and since there was not enough vaccine to treat the entire population, the vaccine was predominantly used in the identified sub-population.

In many cases, however, there is no clearly identifiable sub-population on which to focus control efforts. Furthermore, often the available resources will be insufficient to target all individuals of a sub-population, even if such a group can be identified. As a result, it is critical that the resources are administered in a time-optimal fashion. In other words, if we do not have enough resources to target all appropriate individuals during the entire course of an outbreak, how then should these resources be used over time so as to achieve some specific goal (e.g., minimizing outbreak size)? Such time-optimal control strategies are the focus of this paper.

Although an analysis of the time-optimal application of outbreak controls is of clear practical value, surprisingly little analysis has been done for the basic SIR model (Abakuks 1972, 1973, 1974; Behncke 2000; Greenhalgh 1988; Morton and Wickwire 1974; Wickwire 1975, 1977). In addition, many of the previous results that have been published have not been fully assimilated by practicing epidemiologists and public health officials. Some of the earliest work in this area is by Abakuks. In Abakuks (1973), Abakuks investigated the optimal control of a simple deterministic SIR model, and determined the isolation strategy that minimizes the total number of infected individuals, balanced against a cost associated with using isolation. $\mathrm{He}$ assumed that, at any instant, any number of infectives could be isolated. His results then demonstrated that the optimal control was to either isolate all of the infectives at the beginning of the epidemic, or to never isolate any of them, depending on how heavily the cost of isolation was weighted in the objective function. In Abakuks (1972), Abakuks determined the optimal vaccination strategy for the same model and found that the optimal strategy was to vaccinate $N$ susceptibles at the start of the epidemic, where $N$ depends on the precise form of the objective function. In Abakuks (1974), Abakuks then determined the optimal vaccination strategy for the same model but under the assumption that, at any instant, either all or none of the susceptibles are vaccinated. He found that the optimal control had the same basic form as the optimal control for the isolation model. Shortly after the publication of Abakuks (1973, 1974), Wickwire published Morton and Wickwire (1974) and Wickwire (1975) where he studied the same questions but with two notable differences. Firstly, he removed the unrealistic assumption that an arbitrary number of individuals can be isolated or vaccinated instantaneously, and instead modeled these processes under the assumption that some finite rate of isolation or vaccination is possible. Secondly, Wickwire determined the optimal control that minimizes the total 
infectious burden over an outbreak (plus a cost for using the control) rather than the control that minimizes the total outbreak size. He found that the optimal isolation strategy was to use either maximal control for the entire epidemic or to use no control at all and that the optimal vaccination strategy has at most one switch (switching from maximal vaccination to no vaccination). Wickwire's results therefore, agree qualitatively with Abakuks' results when the control dynamics are changed from acting instantaneously to acting through a rate parameter. Interestingly, however, neither Abakuks nor Wickwire provided results for a combined isolation-vaccination model. In fact, even in Behncke (2000), which expands Wickwire's results to models with more general contact rates, there is no solution provided for such mixed models.

Our work extends that of Abakuks (1972, 1973, 1974), Morton and Wickwire (1974), and Wickwire (1975) by examining the kind of resource constraints mentioned earlier. Specifically, the simple SIR model with mass action contact is revisited, and the following question is asked: "Given that there is a limited amount of control resources available during an outbreak, what control policy minimizes the total outbreak size?" Although such SIR models are probably too simplistic for many real outbreaks of interest for which there is limited control resources, we focus on this simple case for two reasons. First, we are able to provide a complete analytical solution for these cases, both for independent isolation and vaccination, as well as for a mixed model that allows both controls simultaneously. Thus, although these models are not intended as definitive solutions to real-world optimal isolation and vaccination problems, they provide a foundation of rigorous mathematical results upon which more complex models can be built, and for which such complete solutions are not possible. Second, even in this very simple epidemiological model, the analysis is not trivial, and the results have some surprising and initially counterintuitive features. For example, the optimal isolation strategy exhibits threshold behaviour, the solution switching from being non-unique to being unique under different parameter values, whereas the optimal vaccination strategy has a consistent basic form. Secondly, in the case of limited resources, the solution for the optimal control strategy of the mixed model is not, in fact, a direct combination of the optimal isolation and vaccination strategies on their own.

The remainder of this article is structured as follows. Section 2 reviews the basic model used in all further analysis and gives a precise mathematical statement of the problem. Section 3 describes the optimal isolation, optimal vaccination and optimal mixed polices under limited resources. The next three sections then present the proofs for the optimal isolation, optimal vaccination and optimal mixed polices, respectively. Lastly, Sect. 7 discusses some of the salient features of the results.

\section{Model and setup}

The following standard deterministic SIR model is used throughout this article (Anderson and May 1991) 


$$
\begin{aligned}
& \dot{S}=-\beta S I-u_{v} S, \\
& \dot{I}=\beta S I-\left(\mu+u_{i}\right) I,
\end{aligned}
$$

where $S$ and $I$ are the numbers of susceptible and infected hosts (a dot indicating time derivatives), $\beta$ is the transmission rate, $\mu$ is the per capita loss rate of infected individuals through both mortality and recovery, and $u_{v}$ and $u_{i}$ are the per capita rates of vaccination and isolation respectively. Note that, by definition, vaccination has a direct effect only on susceptible individuals whereas isolation has a direct effect only on infected individuals. If $u_{v} \equiv 0$ then (1) describes the standard isolation model, if $u_{i} \equiv 0$ then (1) describes the standard vaccination model, and if neither $u_{v}$ nor $u_{i}$ are the zero function then (1) describes the isolation-vaccination model (or mixed model). Note that we do not explicitly track the dynamics of the recovered sub-population.

The main reason we have chosen to analyze model (1) is that it allows us to extend the work of Abakuks (1972, 1973, 1974), Morton and Wickwire (1974), and Wickwire (1975) to the case when there are limited resources. This being said, it is worth mentioning some of the major assumptions implicit in model (1). The first major assumption is that disease transmission is well modelled using mass action incidence. Although it is perhaps more common to use standard incidence when modeling human disease, there are important studies that use mass action incidence [for example a recent study for influenza, Lipsitch et al. (2007)]. We have therefore decided to use mass action incidence because it is consistent with the models used in Abakuks $(1972,1973,1974)$, Morton and Wickwire (1974), and Wickwire (1975) and because there is a precedent for it in current literature. The second major assumption is that isolation and vaccination are completely effective. In other words, isolated individuals cannot spread the infection and vaccinated individuals cannot contract the infection. Although we have not undertaken the detailed analysis required to fully understand the implications of these assumptions, we have performed some preliminary numerical analysis that suggests that these assumptions do not significantly alter the main conclusions of our work (see Appendix).

Before proceeding further, it is worth discussing the bounds on the control variables, $u_{i}$ and $u_{v}$ in (1). The variables $u_{i}$ and $u_{v}$ are the per-capita rates of isolation and vaccination respectively, and therefore in practice each of these will be related to the isolation and vaccination effort that is employed. For example, the level of $u_{i}$ that can be attained at any given time will, to some extent, depend upon the public health infrastructure (e.g., number of public health officers available). At the same time, however, the characteristics of the disease will impose an upper limit on the possible values of $u_{i}$. For example, if some infections are asymptomatic (or if there is an asymptomatic phase), then even if we employed an infinite isolation effort, $u_{i}$ would remain bounded. This simply reflects the fact that, regardless of how much we strive to increase the rate of isolation or vaccination, it is usually unavoidable that the targeted individuals will spend some nonzero amount of time circulating in the population before they are recruited (i.e., before they are isolated of vaccinated). Therefore, we model this by specifying an upper bound for both control variables, and suppose that this upper bound is disease-specific. The presence of these upper bounds on the control variables is distinct from our assumption (to be formalized shortly) that control resources are limited. The upper bounds reflect unavoidable constraints on the rate at which we can 
isolate or vaccinate, whereas the assumption of limited resources reflects constraints on the total number of people we can isolate or vaccinate.

\section{Remark 1}

(i) Let $z(t)$ denote the total number of vaccinated susceptibles at time $t$ and let $w(t)$ denote the total number of isolated individuals at time $t$. The actual values of $S, I, z$ and $w$ will depend on the specific choice of controls $u_{i}$ and $u_{v}$. When necessary, in order to make this dependence explicit, $S_{\left[u_{i}, u_{v}\right]}, I_{\left[u_{i}, u_{v}\right]}, z_{\left[u_{i}, u_{v}\right]}$ and $w_{\left[u_{i}, u_{v}\right]}$ will be used to denote the states satisfying (1) with the particular choice of controls, $u_{i}$ and $u_{v}$. Furthermore, if $u_{i} \equiv 0\left(u_{v} \equiv 0\right)$ then $S_{\left[u_{v}\right]}\left(S_{\left[u_{i}\right]}\right)$ will be used instead of $S_{\left[0, u_{v}\right]}\left(S_{\left[u_{i}, 0\right]}\right)$.

(ii) Let $c \in \mathbb{R}$. Using a slight abuse of notation, if $u_{i}(t)=c\left(u_{v}(t)=c\right)$ for all time then $u_{i}\left(u_{v}\right)$ will be denoted by $u_{i}=c\left(u_{v}=c\right)$.

The general question addressed in this paper can be phrased mathematically as follows:

\section{General problem}

Fix $w_{\max } \geq 0$ and $z_{\max } \geq 0$. Determine the control for model (1) that minimizes

$$
\int_{t_{0}}^{T} \beta S_{\left[u_{i}, u_{v}\right]} I_{\left[u_{i}, u_{v}\right]} \mathrm{d} t,
$$

subject to $S\left(t_{0}\right)=S_{0}, I\left(t_{0}\right)=I_{0}, T=\inf \left\{t \mid I_{\left[u_{i}, u_{v}\right]}(t)=0.5\right\},\left(u_{i}(t), u_{v}(t)\right) \in$ $\left[0, u_{m_{i}}\right] \times\left[0, u_{m_{v}}\right]$ for all $t \in[0, T]$, and subject to the resource constraints

$$
\int_{t_{0}}^{T} u_{i} I_{\left[u_{i}, u_{v}\right]} \mathrm{d} t \leq w_{\max },
$$

and

$$
\int_{t_{0}}^{T} u_{v} S_{\left[u_{i}, u_{v}\right]} \mathrm{d} t \leq z_{\max } .
$$

All of the results presented here hold for any $u_{m_{i}} \in(0, \infty)$ and $u_{m_{v}} \in(0, \infty)$ but to simplify notation it is assumed that $u_{m_{i}}=u_{m_{v}}=u_{\max }$. Expression (2) represents the total outbreak size over the course of the epidemic, and expressions (3) and (4) reflect the assumption that there is a maximum total amount of isolation and vaccination, respectively, that can be used during the course of the epidemic.

The method of choosing $T$ requires some further explanation. The end of the epidemic in models such as system (1) is not well defined, because the number of infected individuals approaches zero asymptotically as time approaches infinity. As a result, it is not immediately clear how best to choose $T$. The three most natural choices are 
(i) $T=\infty$,

(ii) $T=T_{\max }$, where $T_{\max }$ is a sufficiently large constant, and

(iii) $T=\inf \left\{t \mid I(t)=I_{\min }\right\}$, where $I_{\min }$ is some constant chosen to indicate the end of the epidemic.

A potential problem with choosing methods $\mathrm{i}$ and ii is that the structure of the optimal control can be affected by the dynamics of the system at very large values of $t$ in undesirable ways. For example, suppose $u_{i}$ is nonzero and then at some time $t_{1}$, $I\left(t_{1}\right)<1$ and $w\left(t_{1}\right)=w_{\max }$. Then because constraint (3) is now active, $u_{i}(t)=0$ for all $t>t_{1}$. If $\dot{I}\left(t_{1}^{+}\right)>0$, however, then there will be another peak in infectives before the epidemic settles down permanently. This sort of behaviour is undesirable because the final peak in infectives is caused by a fractional number of infectives. Furthermore, an additional complication of method ii is that it is not clear how large $T$ must be in order to coincide with a reasonable interpretation of the end of the epidemic. Method iii avoids both of these difficulties because it terminates the epidemic as soon as $I(t)=I_{\min }$. In the general problem statement above, $I_{\min }$ was chosen to be 0.5 but any value smaller than 1 would work just as well.

In order to make the solution to the above General Problem more transparent, we break it down into three separate problems:

Problem 1 (Isolation Only) Solve General Problem with $z_{\max }=0$.

Problem 2 (Vaccination Only) Solve General Problem with $w_{\max }=0$.

Problem 3 (Mixed Model) Solve General Problem with $z_{\max }>0$ and $w_{\max }>0$.

The primary method applied to solve these problems is Pontryagin's maximum principle (PMP). (See Berkovitz 1974; Kamien and Schwartz 2003; Pontryagin et al. 1964, for details of the PMP.) A simple application of Filippov's theorem, Agrachev and Sachkov (2004), shows that optimal controls exist for Problems 1, 2 and 3. Therefore, in the sequel the existence of optimal controls will always be assumed.

Finally, it is worthwhile to point out that although the General Problem minimizes the total number of infectives, $\int_{t_{0}}^{T} \beta I S \mathrm{~d} t$, this is equivalent to minimizing the total infectious burden $\int_{t_{0}}^{T} I \mathrm{~d} t$. For Problem $1, \int_{t_{0}}^{T} \beta I S \mathrm{~d} t=S_{0}-S(T)$ and $\int_{t_{0}}^{T} I \mathrm{~d} t=$ $-\frac{1}{\beta} \ln \left(\frac{S(T)}{S_{0}}\right)$, and therefore both quantities are minimized by maximizing $S(T)$. For Problem 2, notice that $\int_{t_{0}}^{T} \beta I S \mathrm{~d} t=I(T)-I_{0}+\mu \int_{t_{0}}^{T} I \mathrm{~d} t$. Since $I(T)$ and $I_{0}$ are fixed, minimizing the total number of infectives and minimizing the total infectious burden are equivalent. For Problem 3 it is also the case that both cost functions lead to the same optimal control. Although this is perhaps less obvious, it can be proven by using the PMP to solve Problem 3 with (2) replaced by $\int_{t_{0}}^{T} I \mathrm{~d} t$. The procedure to do this is very similar to the solution of Problem 3 and so is not detailed further.

\section{Optimal policies}

The following three theorems provide solutions to Problems 1-3. 
Theorem 1 (Optimal Isolation Policy) If $w_{\left[u_{\max }\right]}(T) \leq w_{\max }$, then the optimal isolation policy for Problem 1 is $u_{i}^{*} \equiv u_{\max }$. If $w_{\left[u_{\max }\right]}(T)>w_{\max }$, then the optimal isolation policy $u_{i}^{*}$ is any control $u_{i}$ such that $w_{\left[u_{i}\right]}(T)=w_{\max }$.

A key feature affecting the optimal isolation policy in Theorem 1 is whether or not the resources are sufficient to maintain a maximal isolation rate for the entire duration of the outbreak (i.e., $w_{\left[u_{\max }\right]}(T) \leq w_{\max }$ versus $w_{\left[u_{\max }\right]}(T)>w_{\max }$ ). Whether or not this is the case will depend on several factors. First, it is clear that the disease-specific transmissibility, $\beta$, as well as the per-capita removal rate, $\mu$, will affect whether or not the resources are sufficient. Less obvious, however, is that the maximum possible isolation rate, $u_{\max }$, will also play a role. For example, if $u_{\max }$ is relatively large, then it might be possible to reduce the size of the outbreak substantially, thereby requiring a smaller overall total amount of resources. On the other hand, if $u_{\max }$ is relatively small, then a large outbreak might be unavoidable, meaning that a much larger total pool of resources would be required to maintain a maximal isolation effort for the entire epidemic. Thus, the way the disease in question affects the value of $u_{\max }$ (as well as how the existing public health infrastructure affects $u_{\max }$ ) will play an important role in deciding which of the two outcomes in Theorem 1 occur.

Theorem 1 says that if there are sufficient isolation resources, then the optimal strategy is to isolate infectives with maximal effort for the entire epidemic. If there are not sufficient resources to do this, then the optimal strategy is any strategy that uses all of the available isolation resources. So, for example, it is optimal to start isolating with maximal effort at the start of the epidemic and to continue isolating with maximal effort until all of the resources have run out, but this is only one of many possible optimal strategies.

This non-uniqueness of the optimal solution deserves further consideration as it gives policy makers much freedom in choosing how to isolate individuals. One complication is that exercising this freedom requires knowing a priori that the available resources are insufficient. Data from previous epidemics as well as case reports of the current epidemic can be used to estimate the required resources. Alternatively, in very serious situations, it might be quite obvious that the available resources are insufficient. At the same time, however, this flexibility means that policy makers also have the freedom to optimize other cost functions in addition to (2). For example, if one wanted to minimize the average number of infected individuals per unit time over the entire outbreak, it is shown in the Appendix that the optimal policy is to isolate with maximal effort as long as possible. Given this is also an optimal strategy for the cost function (2), it will simultaneously optimize both criteria.

Theorem 2 (Optimal Vaccination Policy) There exists a $\tau \in[0, T]$ such that the optimal vaccination policy for Problem 2 is

$$
u_{v}^{*}(t)= \begin{cases}u_{\max } & t \in[0, \tau) \\ 0 & t \in[\tau, T]\end{cases}
$$

where $\int_{t_{0}}^{\tau} u_{\max } S_{\left[u_{\max }\right]} \mathrm{d} t=z_{\max }$ if $\tau<T$. That is, the optimal vaccination policy is to vaccinate with maximal effort until either all of the resources are used up or the epidemic is over. 
Theorem 2 reveals that the optimal vaccination policy is unique, with the strategy being to employ maximal vaccination from the beginning of the outbreak, for as long as possible. An interesting consequence of the above two theorems is that, under certain circumstances, the optimal isolation policy is less sensitive to initial conditions than the optimal vaccination policy. For example, if there are insufficient resources to isolate maximally for the entire epidemic then there is no penalty for delaying an isolation program provided the maximal possible number of individuals are still isolated. This is not, however, true for vaccination.

Theorem 3 (Optimal Mixed Policy) There exists a $\tau \in[0, T]$ such that the optimal mixed isolation and vaccination strategy for Problem 3 has one of the following forms:

(i)

$$
\left(u_{i}^{*}(t), u_{v}^{*}(t)\right)= \begin{cases}\left(u_{\max }, u_{\max }\right) & t \in[0, \tau] \\ \left(0, u_{v}^{*}(t)\right) & t \in(\tau, T],\end{cases}
$$

(ii)

$$
\text { where } \int_{t_{0}}^{\tau} u_{\max } I_{\left[u_{\max }, u_{\max }\right]} \mathrm{d} t=w_{\max } \text { if } \tau<T \text {, or }
$$

$$
\left(u_{i}^{*}(t), u_{v}^{*}(t)\right)= \begin{cases}\left(u_{\max }, u_{\max }\right) & t \in[0, \tau] \\ \left(u_{i}^{*}(t), 0\right) & t \in(\tau, T],\end{cases}
$$

$$
\text { where } \int_{t_{0}}^{\tau} u_{\max } S_{\left[u_{\max }, u_{\max }\right]} \mathrm{d} t=z_{\max } \text { if } \tau<T .
$$

In Eq. 6, if $t>\tau$ then $u_{v}^{*}(t)$ represents the optimal control for the corresponding vaccination only model. That is, for $t>\tau, u_{v}^{*}(t)$ is the solution to Problem 2 with $\tilde{z}_{\max }=z_{\max }-\int_{t_{0}}^{\tau} u_{\max } S_{\left[u_{\max }, u_{\max }\right]} \mathrm{d} t$ and initial conditions $\tilde{t}_{0}=\tau, \tilde{S}_{0}=S_{\left[u_{\max }, u_{\max }\right]}(\tau)$ and $\tilde{I}_{0}=I_{\left[u_{\max }, u_{\max }\right]}(\tau)$. In Eq. 7, $u_{i}^{*}(t)$ for $t>\tau$ is defined similarly.

Theorem 3 says that the optimal isolation-vaccination strategy is to isolate and vaccinate with maximal effort until either the vaccination or isolation resources run out. If the isolation resources run out first, then it is optimal to continue with the optimal vaccination strategy. If the vaccination resources run out first, then it is optimal to continue with the optimal isolation strategy. The optimal mixed policy is particularly interesting because it is not simply a combination of the optimal isolation and the optimal vaccination policies. Consider the case where there are insufficient isolation resources to isolate with maximal effort for the entire epidemic. Then, unlike the optimal isolation policy, $u_{i}^{*}$ is no longer given by any control that uses all of the isolation resources. In fact the isolation effort must be maximal at the start of the epidemic and must remain maximal until either the isolation or the vaccination resources run out. If the vaccination resources run out first, then the isolation policy for the remainder of the outbreak can then be anything that uses the remaining isolation resources. 


\section{Proof for optimal isolation policy}

The isolation model with limited resources is described by the following set of equations:

$$
\begin{aligned}
\dot{S} & =-\beta S I, \\
\dot{I} & =\beta S I-\left(\mu+u_{i}\right) I, \\
\dot{w} & =u_{i} I .
\end{aligned}
$$

Phrasing Problem 1 as a maximization problem, the PMP provides the following relations:

The Hamiltonian is

$$
\begin{aligned}
H(t) & =-\lambda_{0} \beta S I-\lambda_{S} \beta S I+\lambda_{I} \beta S I-\lambda_{I}\left(\mu+u_{i}\right) I+\lambda_{w} u_{i} I \\
& =-\dot{\lambda}_{I} I=-\dot{\lambda}_{S} S-\lambda_{I} \mu+\left(\lambda_{w}-\lambda_{I}\right) u_{i} I=0,
\end{aligned}
$$

where the adjoint variables satisfy,

$$
\begin{aligned}
& \dot{\lambda}_{S}=-\left(\lambda_{I}-\lambda_{0}-\lambda_{S}\right) \beta I \\
& \dot{\lambda}_{I}=-\left(\lambda_{I}-\lambda_{0}-\lambda_{S}\right) \beta S-\left(\lambda_{w}-\lambda_{I}\right) u_{i}+\lambda_{I} \mu, \\
& \dot{\lambda}_{w}=0
\end{aligned}
$$

The transversality conditions are $\left(\lambda_{0}, \lambda_{S}(T), \lambda_{I}(T), \lambda_{w}\right)=\left(\lambda_{0}, 0, \lambda_{I}(T), q\right)$, where $q \leq 0$. The optimal control therefore satisfies

$$
u_{i}^{*}= \begin{cases}u_{\max } & \lambda_{w}>\lambda_{I} \\ ? & \lambda_{w}=\lambda_{I} \\ 0 & \lambda_{w}<\lambda_{I}\end{cases}
$$

From Eq. 10 we have $\dot{\lambda}_{I}=0$, and therefore the optimal control is either $u_{i}^{*} \equiv 0$, $u_{i}^{*} \equiv u_{\max }$ or $u_{i}^{*}$ is singular.

The, solution to Problem 1 uses the following three observations:

(i) Without the constraint (3) the solution to Problem 1 is $u_{i}^{*} \equiv u_{\max }$. In the sequel this solution will be called the unconstrained optimal control. The proof of this result is given in the Appendix.

(ii) The total number of isolated individuals can be written as (see Appendix for details):

$$
\int_{t_{0}}^{T} u_{i} I_{\left[u_{i}\right]} \mathrm{d} t=S_{0}-S_{\left[u_{i}\right]}(T)+I_{0}-I_{\left[u_{i}\right]}(T)+\frac{\mu}{\beta} \ln \left(\frac{S_{\left[u_{i}\right]}(T)}{S_{0}}\right)
$$

Equation 13 shows that the constraint value, $w_{\left[u_{i}\right]}(T)=\int_{t_{0}}^{T} u_{i} I_{\left[u_{i}\right]} \mathrm{d} t$, depends only on $S_{\left[u_{i}\right]}(T)$. 
(iii) The cost function can be rewritten as $\int_{t_{0}}^{T} \beta I_{\left[u_{i}\right]} S_{\left[u_{i}\right]} \mathrm{d} t=S_{0}-S_{\left[u_{i}\right]}(T)$ and therefore minimizing the cost function is equivalent to maximizing $S_{\left[u_{i}\right]}(T)$.

Clearly, if $w_{\left[u_{\max }\right]}(T) \leq w_{\max }$ then the optimal control is the unconstrained optimal control $u_{i}^{*} \equiv u_{\max }$. To determine the optimal control when $w_{\left[u_{\max }\right]}(T)>w_{\max }$ first notice that Eq. 13 can be rewritten as

$$
\frac{\mu}{\beta} \ln \left(S_{\left[u_{i}\right]}(T)\right)-S_{\left[u_{i}\right]}(T)=I_{\left[u_{i}\right]}(T)-I_{0}-S_{0}+\frac{\mu}{\beta} \ln \left(S_{0}\right)+w_{\left[u_{i}\right]}(T) .
$$

There are two possible scenarios:

(i) Suppose $w_{\left[u_{\max }\right]}(T)>w_{\max }$ and $S_{\left[u_{\max }\right]}(T)<\frac{\mu}{\beta}$. Then $S_{\left[u_{i}\right]}(T)$ is an increasing function of $w_{\left[u_{i}\right]}(T)$ for any $w_{\left[u_{i}\right]}(T) \leq w_{\max }<w_{\left[u_{\max }\right]}(T)$. Therefore, $u_{i}^{*}$ is any control that uses all of the available resources.

(ii) Suppose $w_{\left[u_{\max }\right]}(T)>w_{\max }$ and $S_{\left[u_{\max }\right]}(T)>\frac{\mu}{\beta}$. Since $f(S)=\frac{\mu}{\beta} \ln (S)-S$ is a convex down function with maximum $S=\frac{\mu}{\beta}$, for any $u_{i}$ satisfying $w_{\left[u_{i}\right]}(T)<$ $w_{\max }$ it must be that $S_{\left[u_{i}\right]}(T)<\frac{\mu}{\beta}$. Then $S_{\left[u_{i}\right]}(T)$ is an increasing function of $w_{\left[u_{i}\right]}(T)$ for any $w_{\left[u_{i}\right]}(T)<w_{\max }$. Therefore, $u_{i}^{*}$ is any control that uses all of the available resources.

This concludes the proof of Theorem 1 .

\section{Proof for optimal vaccination policy}

The vaccination model with limited resources is described by the following set of equations:

$$
\begin{aligned}
\dot{S} & =-\beta S I-u_{v} S, \\
\dot{I} & =\beta S I-\mu I, \\
\dot{z} & =u_{v} S .
\end{aligned}
$$

Phrasing Problem 2 as a maximization problem, the PMP provides the following relations:

The Hamiltonian is

$$
\begin{aligned}
H(t) & =-\lambda_{0} \beta S I-\lambda_{S} \beta S I-\lambda_{S} u_{v} S+\lambda_{I} \beta S I-\lambda_{I} \mu I+\lambda_{z} u_{v} S, \\
& =-\dot{\lambda}_{I} I+\left(\lambda_{z}-\lambda_{S}\right) u_{v} S=-\dot{\lambda}_{S} S-\lambda_{I} \mu I=0,
\end{aligned}
$$

where the adjoint variables satisfy,

$$
\begin{aligned}
& \dot{\lambda}_{S}=-\left(\lambda_{I}-\lambda_{0}-\lambda_{S}\right) \beta I-\left(\lambda_{z}-\lambda_{S}\right) u_{v}, \\
& \dot{\lambda}_{I}=-\left(\lambda_{I}-\lambda_{0}-\lambda_{S}\right) \beta S+\lambda_{I} \mu, \\
& \dot{\lambda}_{z}=0 .
\end{aligned}
$$


The transversality conditions are $\left(\lambda_{0}, \lambda_{S}(T), \lambda_{I}(T), \lambda_{z}\right)=\left(\lambda_{0}, 0, \lambda_{I}(T), p\right)$, where $p \leq 0$. The optimal control therefore satisfies

$$
u_{v}^{*}= \begin{cases}u_{\max } & \lambda_{z}>\lambda_{S} \\ ? & \lambda_{z}=\lambda_{S} \\ 0 & \lambda_{z}<\lambda_{S} .\end{cases}
$$

The first step is to show that the optimal control is purely bang-bang (that is, has no singular components). If $\lambda_{z}=\lambda_{S}$ on some interval $J$ then $\dot{\lambda}_{S}=0$ on $J$. Equation 15 then gives

$$
\begin{aligned}
0 & =-\left(-\lambda_{0}-\lambda_{S}+\lambda_{I}\right) \beta I-\left(\lambda_{z}-\lambda_{S}\right) u_{v}, \\
0 & =-\left(-\lambda_{0}-\lambda_{S}+\lambda_{I}\right) \beta I, \\
0 & =\left(-\lambda_{0}-\lambda_{S}+\lambda_{I}\right), \\
\lambda_{I} & =\lambda_{0}+\lambda_{S},
\end{aligned}
$$

and therefore $\dot{\lambda}_{I}=0$ on $J$. If $\dot{\lambda}_{I}=0$, then by Eqs. 16 and 18 it must be that $\lambda_{I}=0$. Therefore $\lambda_{S}=-\lambda_{0}$ and the only nonzero choice for the adjoint variables on $J$ is $\left(\lambda_{0}, \lambda_{S}, \lambda_{I}, \lambda_{z}\right)=(1,-1,0,-1)$. Further, by Eqs. 15 and 16, once $u_{v}^{*}$ becomes singular then it must remain singular (that is, $T \in J$ ). Now since $T \in J$, $\left(\lambda_{0}, \lambda_{S}, \lambda_{I}, \lambda_{z}\right)=(1,-1,0,-1)$ must satisfy the transversality condition $\lambda_{S}(T)=0$. Since the transversality condition is not satisfied, the optimal control must be purely bang-bang.

Next, we consider the times at which the optimal control switches between 0 and $u_{\text {max }}$. Suppose the optimal control switches at times $t_{s_{i}}$. Then

$$
H\left(t_{s_{i}}\right)=-\dot{\lambda}_{I}\left(t_{s_{i}}\right) I\left(t_{s_{i}}\right)=-\dot{\lambda}_{S}\left(t_{s_{i}}\right) S\left(t_{s_{i}}\right)-\lambda_{I}\left(t_{s_{i}}\right) \mu I\left(t_{s_{i}}\right)=0 .
$$

Therefore, substituting $\dot{\lambda}_{I}\left(t_{s_{i}}\right)=0$ into Eq. 16 gives

$$
\left(\lambda_{S}\left(t_{s_{i}}\right)+\lambda_{0}\right) \beta S\left(t_{s_{i}}\right)=\lambda_{I}\left(t_{s_{i}}\right)\left(\beta S\left(t_{s_{i}}\right)-\mu\right) .
$$

The rest of the proof relies on the following relations which are obtained from Eq. 17 and Eq. 19:

$$
\begin{aligned}
& \lambda_{I}\left(t_{s_{i}}\right)>0 \Rightarrow \dot{\lambda}_{S}\left(t_{s_{i}}\right)<0 \Rightarrow\left(0 \rightarrow u_{\max }\right) \\
& \lambda_{I}\left(t_{s_{i}}\right)<0 \Rightarrow \dot{\lambda}_{S}\left(t_{s_{i}}\right)>0 \Rightarrow\left(u_{\max } \rightarrow 0\right) \\
& \lambda_{I}\left(t_{s_{i}}\right)=0 \Rightarrow \dot{\lambda}_{S}\left(t_{s_{i}}\right)=0 \Rightarrow \text { no switch occurs. }
\end{aligned}
$$


Remark 2 In (21), the notation $(a \rightarrow b)$ means that $u_{v}^{*}$ switches from the value $a$ to the value $b$ at $t=t_{s_{i}}$. In the sequel, the notation:

$$
\begin{aligned}
& a \rightarrow b \rightarrow b \\
& c \rightarrow d \rightarrow e
\end{aligned}
$$

describes two possible forms for $u_{v}^{*}$. In the first form, $u_{v}^{*}$ has the value $a$ for some time and then switches to the value $b$ for the remainder of the time. In the second form, $u_{v}^{*}$ has the value $c$ for some time and then switches to the value $d$ for some time and then switches to the value $e$ for the remainder of the time.

Now since $\lambda_{S}\left(t_{s_{i}}\right)=\lambda_{z}=$ constant, $\lambda_{S}\left(t_{s_{i}}\right)+\lambda_{0}$ is either always positive, always negative or always zero.

Suppose $\lambda_{S}\left(t_{s_{i}}\right)+\lambda_{0}=0$. Then by (20), either $\lambda_{I}\left(t_{s_{i}}\right)=0$ or $S\left(t_{s_{i}}\right)=\frac{\mu}{\beta}$. By (21), if $\lambda_{I}\left(t_{s_{i}}\right)=0$ then no switch occurs. Alternatively if $S\left(t_{s_{i}}\right)=\frac{\mu}{\beta}$ then the optimal control has only one switch and this switch occurs when $I$ is maximal. The possible optimal controls are:

$$
\begin{aligned}
u_{\max } & \rightarrow u_{\max } \\
u_{\max } & \rightarrow 0 \\
0 & \rightarrow u_{\max } \\
0 & \rightarrow 0
\end{aligned}
$$

Next consider the case when $\lambda_{S}\left(t_{s_{i}}\right)+\lambda_{0}>0$. By Eq. 20, if $\lambda_{S}\left(t_{s_{i}}\right)+\lambda_{0}>0$ then either

(i) $\lambda_{I}\left(t_{s_{i}}\right)>0$ and $S\left(t_{s_{i}}\right)>\frac{\mu}{\beta}$ or

(ii) $\lambda_{I}\left(t_{s_{i}}\right)<0$ and $S\left(t_{s_{i}}\right)<\frac{\mu}{\beta}$.

Suppose that at $t_{s_{1}}$ possibility i holds. Then by (21), $u_{v}^{*}$ switches from 0 to $u_{\max }$ at $t_{s_{1}}$. If there is to be another switch (say at $t=t_{S_{2}}$ ) then it must be from $u_{\max }$ to 0 . This implies, by (21), that ii must hold at $t_{s_{2}}$. If there is to be a third switch (say at $t=t_{s_{3}}$ ) then it must be from 0 to $u_{\max }$ and therefore, by (21), i must hold at $t_{s_{3}}$. But this is not possible because $S$ is a monotonically decreasing function of time. Similar reasoning applies to the case when possibility ii holds at $t_{s_{1}}$. Therefore, if $\lambda_{S}\left(t_{s_{i}}\right)+\lambda_{0}>0$ the optimal control can have at most two switches. The possible forms for the optimal control are:

$$
\begin{aligned}
& u_{\max } \rightarrow u_{\max } \rightarrow u_{\max } \\
& u_{\max } \rightarrow 0 \rightarrow 0 \\
& 0 \rightarrow u_{\max } \rightarrow 0 \\
& 0 \quad \rightarrow u_{\max } \rightarrow u_{\max } \\
& 0 \quad \rightarrow 0 \rightarrow 0
\end{aligned}
$$

Notice however, that the control form $0 \rightarrow u_{\max } \rightarrow 0$ is not optimal. To see this, recall that the Hamiltonian can be written as $H=-\dot{\lambda}_{I} I+\left(\lambda_{z}-\lambda_{S}\right) u_{v} S$ and therefore 
$\dot{\lambda}_{I}(t) \geq 0$ for all $t \in[0, T]$. Also, by $(21), \lambda_{I}\left(t_{s_{1}}\right)>0$ and $\lambda_{I}\left(t_{s_{2}}\right)<0$, but this is not possible since $\lambda_{I}$ is monotonically increasing.

Next consider the case when $\lambda_{S}\left(t_{s_{i}}\right)+\lambda_{0}<0$. Applying similar arguments to those that were used for the case when $\lambda_{S}\left(t_{s_{i}}\right)+\lambda_{0}>0$, the possible forms for the optimal control are:

$$
\begin{aligned}
& u_{\max } \rightarrow u_{\max } \rightarrow u_{\max } \\
& u_{\max } \rightarrow 0 \rightarrow 0 \\
& 0 \quad \rightarrow u_{\max } \rightarrow u_{\max } \\
& u_{\max } \rightarrow 0 \rightarrow u_{\max } \\
& 0 \quad \rightarrow 0 \rightarrow 0
\end{aligned}
$$

Notice however, that the control form, $u_{\max } \rightarrow 0 \rightarrow u_{\max }$ is not optimal because at the first switch $\lambda_{I}\left(t_{s_{1}}\right)<0$ and at the second switch $\lambda_{I}\left(t_{s_{2}}\right)>0$. This implies that $\lambda_{I}$ is non-constant while $u_{v}^{*}=0$ which contradicts Eq. 14 .

Combining the control forms in (22), (23) and (24) gives the following candidates for the optimal control:

$$
\begin{aligned}
& u_{\max } \rightarrow u_{\max } \rightarrow u_{\max } \\
& u_{\max } \rightarrow 0 \rightarrow 0 \\
& 0 \quad \rightarrow u_{\max } \rightarrow u_{\max } \\
& 0 \quad \rightarrow 0 \rightarrow 0
\end{aligned}
$$

The next step is to show that the control forms, $0 \rightarrow u_{\max } \rightarrow u_{\max }$ and $0 \rightarrow 0 \rightarrow 0$, are not optimal. Recall that $\dot{\lambda}_{I} \geq 0$. Therefore, since $\lambda_{I}\left(t_{s_{i}}\right)>0$, it must be that $\lambda_{I}(t)>0$ for all $t \in\left[t_{s_{1}}, T\right]$. Therefore, by Eq. $14, \dot{\lambda}_{S}(t)<0$ for all $t \in\left[t_{s_{1}}, T\right]$. Since $\lambda_{S}(T)=0$ this implies that $\lambda_{S}(t)>0$ for all $t \in\left[t_{S_{1}}, T\right)$ and therefore $\lambda_{S}\left(t_{s_{1}}\right)=\lambda_{z}>0$ but this is a contradiction since $\lambda_{z} \leq 0$. The proof that $u_{v} \equiv 0$ is not optimal is given in the Appendix.

The discussion above shows that the optimal control is given by,

$$
u^{*}(t)= \begin{cases}u_{\max } & t \in\left[0, t_{s_{1}}\right) \\ 0 & t \in\left[t_{s_{1}}, T\right] .\end{cases}
$$

To prove the assertion about $t_{s_{1}}$, suppose that $u \equiv u_{\max }$ is not optimal. Then, since the optimal control has the form $u_{\max } \rightarrow 0$, by $(21), \lambda_{I}\left(t_{s_{1}}\right)<0$. This implies, since $\lambda_{I}$ is monotonically increasing, that $\lambda_{I}(t)<0$ for all $t \in\left[0, t_{s_{1}}\right]$. This implies, since $\dot{\lambda}_{I}(t)=0$ for all $t \in\left[t_{s_{1}}, T\right]$, that $\lambda_{I}(t)<0$ for all $t \in[0, T]$. By Eq. 14, this shows that $\dot{\lambda}_{S}>0$ for all $t \in[0, T)$ and therefore $\lambda_{S}(t)<0$ for all $t \in[0, T)$ (since $\lambda_{S}(T)=0$ ). Therefore, if $t_{s_{1}}<T$ then $\lambda_{S}\left(t_{s_{1}}\right)=\lambda_{z}<0$. But $\lambda_{z}<0$ only if $z_{\max }=z(T)$ and therefore all of the resources must be used up. 


\section{Proof for optimal mixed policy}

The mixed model with limited resources is described by the following set of equations:

$$
\begin{aligned}
\dot{S} & =-\beta S I-u_{v} S, \\
\dot{I} & =\beta S I-\left(\mu+u_{i}\right) I, \\
\dot{w} & =u_{i} I, \\
\dot{z} & =u_{v} S .
\end{aligned}
$$

Phrasing Problem 3 as a maximization problem, the PMP provides the following relations:

The Hamiltonian is

$$
\begin{aligned}
H(t) & =-\lambda_{0} \beta S I-\lambda_{S} \beta S I-\lambda_{S} u_{v} S+\lambda_{I} \beta S I-\lambda_{I}\left(\mu+u_{i}\right) I+\lambda_{w} u_{i} I+\lambda_{z} u_{v} S, \\
& =-\dot{\lambda}_{I} I+\left(\lambda_{z}-\lambda_{S}\right) u_{v} S=-\dot{\lambda}_{S} S+\left(\lambda_{w}-\lambda_{I}\right) u_{i} I-\lambda_{I} \mu I=0,
\end{aligned}
$$

where the adjoint variables satisfy,

$$
\begin{aligned}
& \dot{\lambda}_{S}=-\left(\lambda_{I}-\lambda_{0}-\lambda_{S}\right) \beta I-\left(\lambda_{z}-\lambda_{S}\right) u_{v}, \\
& \dot{\lambda}_{I}=-\left(\lambda_{I}-\lambda_{0}-\lambda_{S}\right) \beta S-\left(\lambda_{w}-\lambda_{I}\right) u_{i}+\lambda_{I} \mu, \\
& \dot{\lambda}_{z}=0, \\
& \dot{\lambda}_{w}=0 .
\end{aligned}
$$

The transversality conditions are $\left(\lambda_{0}, \lambda_{S}(T), \lambda_{I}(T), \lambda_{z}, \lambda_{w}\right)=\left(\lambda_{0}, 0, \lambda_{I}(T), p, q\right)$, where $p, q \leq 0$. The optimal control therefore satisfies

$$
u_{v}^{*}= \begin{cases}u_{\max } & \lambda_{z}>\lambda_{S} \\ ? & \lambda_{z}=\lambda_{S} \\ 0 & \lambda_{z}<\lambda_{S}\end{cases}
$$

and

$$
u_{i}^{*}= \begin{cases}u_{\max } & \lambda_{w}>\lambda_{I} \\ ? & \lambda_{w}=\lambda_{I} \\ 0 & \lambda_{w}<\lambda_{I}\end{cases}
$$

Remark 3

(i) In the sequel $u_{i}$ and $u_{v}$ will denote the optimal controls (instead of $u_{i}^{*}$ and $u_{v}^{*}$ ). The notation $u_{v}^{*}$ and $u_{i}^{*}$ will denote the optimal controls for the vaccination only model and isolation only model (that is, solutions to Problems 2 and 1 respectively with appropriate choices of $\tilde{t}_{0}, \tilde{z}_{\max }, \tilde{w}_{\max }, \tilde{\mu}, \tilde{I}_{0}$ and $\tilde{S}_{0}$ ).

(ii) In the sequel, the notation:

$$
\begin{aligned}
& u_{i}: a \rightarrow b \rightarrow b \rightarrow \\
& u_{v}: c \rightarrow d \rightarrow e \rightarrow \\
& t: 0 \rightarrow t_{s_{1}} \rightarrow t_{s_{2}} \rightarrow
\end{aligned}
$$


means that there are two possible switch times, $t_{s_{1}}$ and $t_{s_{2}}$, and $u_{i}$ switches from $a$ to $b$ at $t_{s_{1}}$ and does not switch at time $t_{s_{2}}$. Similarly, $u_{v}$ switches from $c$ to $d$ at time $t_{s_{1}}$ and from $d$ to $e$ at time $t_{s_{2}}$.

The solution to Problem 3 uses the following claims (the proofs of which can be found in the Appendix).

Claim 3.1 If $u_{v}$ is singular on some interval $J$ then $u_{i}$ is not singular on $J$ and $u_{i}=0$ on $J$.

Claim 3.2 If $u_{i}$ is singular on an interval $J$, then $u_{v}=0$ on $J$.

Claim 3.3 When $u_{i}$ is singular, denote it by $u_{s}$. If $u_{i}$ has a singular component, then $u_{i}$ has one of the following forms:

$$
\begin{aligned}
u_{\max } & \rightarrow u_{\mathrm{s}} \\
u_{\mathrm{s}} & \rightarrow u_{\mathrm{s}} .
\end{aligned}
$$

Claim 3.4 If there exists a $t_{s} \geq 0$ such that $u_{i}$ is constant for all $t \in\left(t_{s}, T\right]$ then $u_{v}(t)=u_{v}^{*}(t)$ for all $t \in\left(t_{s}, T\right]$.

Claim 3.5 If there exists a $t_{s} \geq 0$ such that $u_{v}(t)=0$ for all $t \in\left(t_{s}, T\right]$ then $u_{i}(t)=u_{i}^{*}(t)$ for all $t \in\left(t_{s}, T\right]$.

Claim 3.6 Let $J$ be a maximal interval such that

(i) $u_{i}=u_{\max }$ on $J$ and

(ii) $u_{i}$ is not singular on $J$.

If $T \notin J$ then $u_{v}=u_{\max }$ on $J$.

Claims 3.1-3.6 and the fact that $\dot{\lambda}_{I} \geq 0$, imply that the optimal control must have one of the following forms:

$$
\begin{aligned}
u_{i} & : u_{s} \rightarrow \\
u_{v} & : 0 \rightarrow \\
t & : 0 \rightarrow, \\
u_{i} & : 0 \rightarrow \\
u_{v} & : u_{v}^{*} \rightarrow \\
t & : 0 \rightarrow, \\
u_{i} & : u_{\max } \rightarrow \\
u_{v} & : u_{v}^{*} \rightarrow \\
t & : 0 \rightarrow, \\
u_{i} & : u_{\max } \rightarrow u_{s} \rightarrow \\
u_{v} & : u_{\max } \rightarrow 0 \rightarrow
\end{aligned}
$$




$$
\begin{aligned}
t & : 0 \rightarrow t_{s_{1}} \rightarrow, \\
u_{i} & : u_{\max } \rightarrow 0 \rightarrow \\
u_{v} & : u_{\max } \rightarrow u_{v}^{*} \rightarrow \\
t & : 0 \rightarrow t_{s_{1}} \rightarrow .
\end{aligned}
$$

Remark 4 In the expressions (29)-(33) the instances when $u_{i}$ can be singular are explicitly indicated. For example if $u_{i}$ has the form specified in (32) then $u_{i}$ is singular for all $t>t_{s_{1}}$. Conversely, if $u_{i}$ has the form specified in (33) then $u_{i}$ is never singular. In other words, form (33) not only specifies that $u_{i}(t)=0$ for all $t>t_{s_{1}}$ but also that $u_{i}$ is not singular.

Claim 3.7 The optimal control is not of the form (29).

Proof If the optimal control is of the form (29) then it is also the optimal control for the isolation only model. Therefore, there exists a $t_{s} \in[0, T]$ such that

$$
u_{i}(t)= \begin{cases}u_{\max } & t \in\left[0, t_{s}\right] \\ 0 & t \in\left(t_{s}, T\right]\end{cases}
$$

is optimal. Now fix this choice of $u_{i}$. The next step is to show that there exists a $u_{v}$ that gives a lower value for the cost function then $u_{v} \equiv 0$ does.

Suppose $t_{s} \neq T$ then, by the principle of optimality, it follows that

$$
\left(u_{i}(t), u_{v}(t)\right)= \begin{cases}\left(u_{\max }, 0\right) & t \in\left[0, t_{s}\right) \\ \left(0, u_{v}^{*}\right) & t \in\left[t_{s}, T\right]\end{cases}
$$

has a smaller cost than a control of the form (29).

Alternatively, suppose $t_{s}=T$, then the mixed model essentially becomes a vaccination only model with new parameter $\tilde{\mu}=\mu+u_{\max }$. Therefore, $u_{v}=u_{v}^{*}$ gives a lower value for the cost function than $u_{v} \equiv 0$ does.

Claim 3.8 If the optimal control is of the form (30) then $u_{v}^{*} \equiv u_{\max }$.

Proof Suppose to the contrary that

$$
u_{v}^{*}(t)= \begin{cases}u_{\max } & t \in\left[0, t_{s}\right] \\ 0 & t \in\left(t_{s}, T\right]\end{cases}
$$

where $t_{s}<T$. Then it follows that

$$
\left(u_{i}(t), u_{v}(t)\right)= \begin{cases}\left(0, u_{\max }\right) & t \in\left[0, t_{s}\right] \\ \left(u_{i}^{*}, 0\right) & t \in\left(t_{s}, T\right]\end{cases}
$$

will give a lower value for the cost function than a control of the form (30) will.

Claim 3.9 The optimal control is not of the form (30). 
Proof If the optimal control is of the form (30) then $\int_{t_{0}}^{T} u_{i} I \mathrm{~d} t=0<w_{\max }$ and therefore $\lambda_{w}=0$. Since $u_{i}$ is not singular (see Remark 4), this implies that $\lambda_{I}(t)>0$ for all $t>t_{0}$. Therefore, by Eq. 27, $\dot{\lambda}_{S}(t)<0$ for all $t>t_{0}$. This implies that $\lambda_{S}(t)>0$ (since $\lambda_{S}(T)=0$ ) for all $t<T$, but this gives a contradiction since if $\lambda_{S}(t)>0$ then $u_{v}(t)=0$.

Claim 3.10 If the optimal control is of the form (32) then $\int_{t_{0}}^{t_{s_{1}}} u_{\max } S_{\left[u_{\max }, u_{\max }\right]} \mathrm{d} t=$ $z_{\max }$.

Proof By Claim 3.5, if the optimal control has the form (32) then $u_{s}=u_{i}^{*}$. Therefore there exists a $t_{s_{2}} \in\left[t_{s_{1}}, T\right]$ such that

$$
u_{i}(t)= \begin{cases}u_{\max } & t \in\left[t_{0}, t_{s_{1}}\right] \\ u_{\max } & t \in\left(t_{s_{1}}, t_{s_{2}}\right] \\ 0 & t \in\left(t_{s_{2}}, T\right]\end{cases}
$$

is optimal. Assume that the number of people vaccinated at $t=t_{s_{1}}$ is not the maximal amount, that is assume $\int_{t_{0}}^{t_{S_{1}}} u_{\max } S_{\left[u_{\max }, u_{\max }\right]} \mathrm{d} t<z_{\max }$. Suppose $t_{S_{2}} \neq T$ then it follows that

$$
\left(u_{i}(t), u_{v}(t)\right)= \begin{cases}\left(u_{\max }, u_{\max }\right) & t \in\left[t_{0}, t_{s_{1}}\right] \\ \left(u_{\max }, 0\right) & t \in\left(t_{s_{1}}, t_{s_{2}}\right] \\ \left(0, u_{v}^{*}\right) & t \in\left(t_{s_{2}}, T\right],\end{cases}
$$

has a smaller cost then a control of the form (32).

Alternatively, suppose $t_{s_{2}}=T$, then the mixed model essentially becomes a vaccination only model with new parameter $\tilde{\mu}=\mu+u_{\max }$. Therefore, $u_{v}=u_{v}^{*}$ is the optimal choice for $u_{v}$ and so if $u_{v}=u_{v}^{*}$ has the form specified in (32) then it must be that $\int_{t_{0}}^{t_{s}} u_{\max } S_{\left[u_{\max }, u_{\max }\right]} \mathrm{d} t=z_{\max }$.

Claim 3.11 If the optimal control is of the form (33) then $\int_{t_{0}}^{t_{s}} u_{\max } I_{\left[u_{\max }, u_{\max }\right]} \mathrm{d} t=$ $w_{\max }$.

Proof The first step is to show that $\lambda_{I}(t) \leq 0$ for all $t \in[0, T)$. Suppose to the contrary that there exists a $t^{*} \in[0, T)$ such that $\lambda_{I}\left(t^{*}\right)$ is positive. Then, since $\dot{\lambda}_{I} \geq 0$, it must be that $\lambda_{I}(t)>0$ for all $t \in J=\left[t^{*}, T\right]$. Therefore, since $\lambda_{w} \leq 0, u_{i}=0$ on $J$. This implies, by Eq. 27, that $\dot{\lambda}_{S}<0$ on $J$ and hence $\lambda_{S}>0$ on $J$ (since $\lambda_{S}(T)=0$ ). Similarly, since $\lambda_{S}>0$ on $J, u_{v}=0$ on $J$ and this implies, by Eq. 27 , that $\dot{\lambda}_{I}=0$ on $J$. Letting $\dot{\lambda}_{I}=0$ and rearranging Eq. 28, gives

$$
\lambda_{I}(\beta S-\mu)=\left(\lambda_{0}+\lambda_{S}\right) \beta S .
$$

Now both the right-hand side of Eq. 34 and $\lambda_{I}$ are positive on $J$. This implies that $S(t)>\frac{\mu}{\beta}$ for all $t \in J$. But this contradicts the fact that $S(T)<\frac{\mu}{\beta}$. This proves that $\lambda_{I}(t) \leq 0$ for all $t \in[0, T)$.

Now, $\dot{\lambda}_{I} \geq 0, \lambda_{I} \leq 0$ and $u_{i}$ switches from $u_{\max }$ to 0 when $\lambda_{I}=\lambda_{w}$. Therefore if $\lambda_{w}=0$ then the switch occurs while $u_{i}$ is singular, since $u_{i}$ is not singular (see Remark 4) it must be that $\lambda_{w}<0$. 
Lastly, notice that control form (31) covers the case when there are enough resources to isolate with maximal effort for the entire epidemic. This proves Theorem 3.

\section{Discussion}

The results presented in Sect. 3 contain many interesting features. Firstly, recall that Wickwire showed that the optimal isolation policy is either to isolate with maximal effort for the entire epidemic or to never isolate any infectives. In contrast, the optimal isolation policy presented in Theorem 1 is to isolate with maximal effort for the entire epidemic only if there are enough resources. If there are not enough resources, the optimal policy is any policy that uses all of the resources. In other words, the optimal isolation policy presented by Wickwire is characteristically different than the one presented here. Conversely, the optimal vaccination policies given in Wickwire (1975) and Theorem 2 have the same basic form. These differences are partially explained by the PMP relations. A simple calculation shows that the basic form of the PMP relations are the same for the case when the objective function includes a term linear in the control and the case when the total amount of resources are limited. Correspondingly, the optimal isolation policy is singular, so the PMP relations are not sufficient to determine the optimal isolation policy. This means that the optimal isolation policies in Morton and Wickwire (1974) and Theorem 1 can be different. For the vaccination model however, the PMP relations are necessary and sufficient.

Another interesting aspect of the isolation only model is that for certain parameter values, small changes in $w_{\max }$ can cause large changes in the value of the minimal cost. To see this, notice that setting $u_{v} \equiv 0$ and $u_{i} \equiv u_{\max }$ in (1) gives,

$$
w_{\left[u_{\max }\right]}(T)=\int_{t_{0}}^{T} u_{\max } I_{\left[u_{\max }\right]} \mathrm{d} t=-\frac{u_{\max }}{\beta} \ln \left(\frac{S_{\left[u_{\max }\right]}(T)}{S_{0}}\right) .
$$

Rearranging Eq. 35 gives, $S_{\left[u_{\max }\right]}(T)=S_{0} \exp \left(\frac{-\beta w_{\left[u_{\max }\right]}(T)}{u_{\max }}\right)$ which expresses the final number of susceptibles for the case when $u_{i} \equiv u_{\max }$, in terms of $w_{\left[u_{\max }\right]}(T)$.

Figure 1 shows that if $S_{\left[u_{\max }\right]}(T)>\frac{\mu}{\beta}$, then as $w_{\max }$ switches from less than $w_{\left[u_{\max }\right]}(T)$ to greater than $w_{\left[u_{\max }\right]}(T)$, there will be a jump in $S_{\left[u_{i}^{*}\right]}(T)$. That is, if $S_{\left[u_{\max }\right]}(T)>\frac{\mu}{\beta}$, then there is a range of values of the final number of susceptible individuals, $S_{\left[u_{i}^{*}\right]}(T)$, that cannot be attained, regardless of the value of $w_{\max }$. Finally, it is worth pointing out that whether or not $S_{\left[u_{\max }\right]}(T)>\frac{\mu}{\beta}$ can be determined directly from the model parameters (without resorting to simulations). To see this, notice that combining Eqs. 13 and 35 shows that $S_{\left[u_{\max }\right]}(T)>\frac{\mu}{\beta}$ if and only if

$$
-\frac{u_{\max }}{\beta} \ln \left(\frac{\mu}{\beta S_{0}}\right)>S_{0}-\frac{\mu}{\beta}+\frac{\mu}{\beta} \ln \left(\frac{\mu}{\beta S_{0}}\right)-I_{T}+I_{0} .
$$

The top panel of Fig. 1 shows a scenario when Eq. 36 is not satisfied and the bottom panel of Fig. 1 shows a scenario when Eq. 36 is satisfied. 

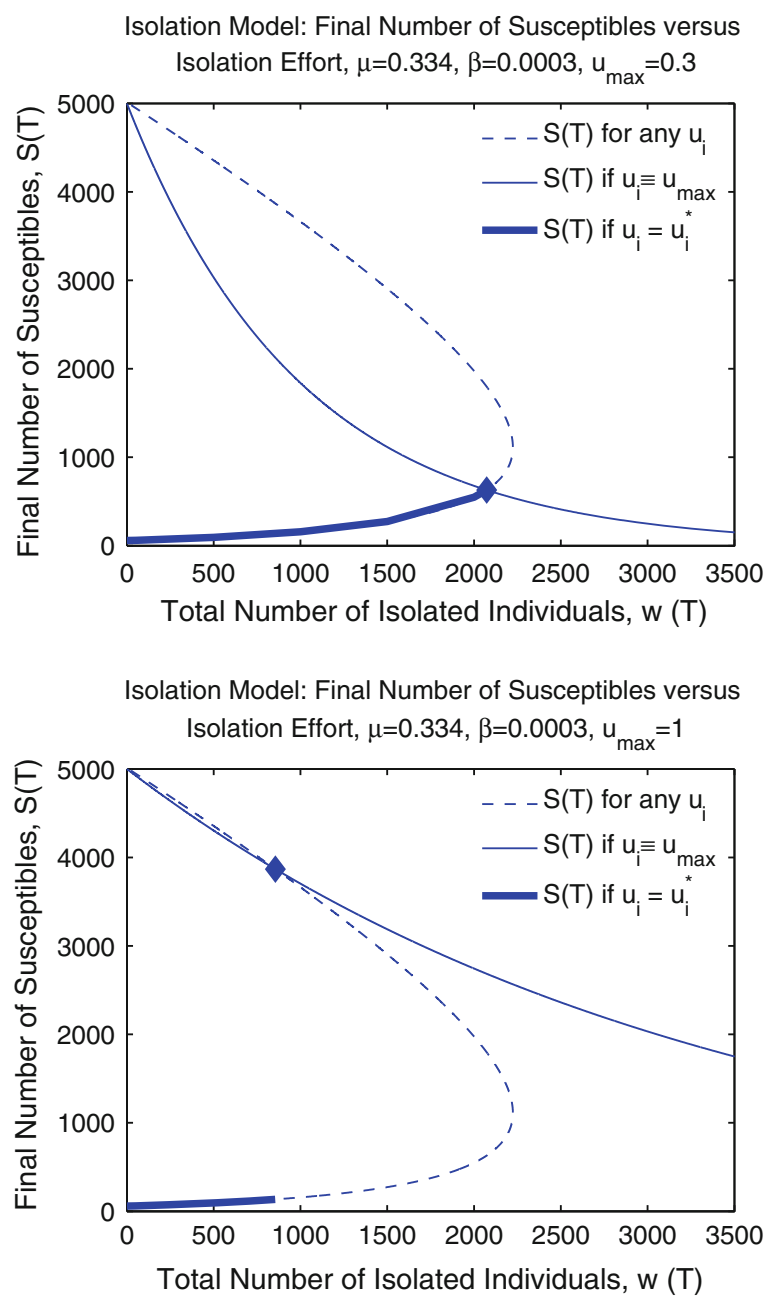

Fig. 1 Top panel: The final number of susceptibles depends only on the total number of isolated individuals (see Eq. 13). For parameter values, $I_{0}=10, S_{0}=5000, u_{\max }=0.3, \mu=0.334$ and $\beta=0.0003$, the dashed curve describes this relationship. The diamond marks the point $\left(w_{\left[u_{\max }\right]}(T), S_{\left[u_{\max }\right]}(T)\right)$. Since $u_{i} \equiv u_{\max }$ is the optimal solution for the unconstrained model and since minimizing the total number of infected is equivalent to maximizing $S(T)$, the diamond marks the largest attainable $S(T)$. The solid thick curve (which lies directly on top of the dashed curve) therefore denotes all possible optimal pairs $(w(T), S(T))$ for our specific choice of parameters. The point marked by the diamond is also the intersection of the dashed curve and the solid thin curve. The solid thin curve plots the final number of susceptibles under the assumption that $u_{i} \equiv u_{\max } . S(T)=\frac{\mu}{\beta}$ at the right-most point of the dashed curve. Since the point $\left(w(T), \frac{\mu}{\beta}\right)$ on the solid thin curve lies to the left of the right-most point on the dashed curve, all optimal values of $S(T)$ must be less than $\frac{\mu}{\beta}$. Bottom panel: This plot shows the same information as the plot on the left-hand side but for the case when $u_{\max }=1$. For this case the intersection of the solid thin curve and the dashed curve occurs at an $S(T)$ larger than $\frac{\mu}{\beta}$. The solid thick curve shows all possible optimal pairs $(w(T), S(T))$ for cases when $w_{\max }<w_{\left[u_{\max }\right]}(T)$. For cases when $w_{\max } \geq w_{\left[u_{\max }\right]}(T)$, the optimal pair is $\left(w_{\left[u_{\max }\right]}(T), S_{\left[u_{\max }\right]}(T)\right)$ (that is, the point marked by the diamond). Therefore, as $w_{\max }$ is increased past the value $w_{\left[u_{\max }\right]}(T)$ the final number of susceptibles jumps from a small value (less than 500) to a large value (greater than 3500) 
The non-uniqueness of $u_{i}^{*}$ when $w_{\max }<w_{\left[u_{\max }\right]}(T)$ means that epidemics with different dynamics can still optimize the cost function of Problem 1. The top panel in Fig. 2 shows three different optimal isolation strategies for a case were $w_{\max }=1450$ and $w_{\left[u_{\max }\right]}(T)=2072$. In all three cases the total infectious burden is 9884.9 and the total number of infected is 4742.4.

The top panel in Fig. 2 shows that very different dynamics result from these three different controls even though all three are optimal. For example the length of the epidemic described by the dotted curve is significantly shorter than the other two epidemics but it reaches a higher peak intensity (as measured by the maximum number of infected). Although the epidemics described by the dashed and solid curves are very similar, the dashed curve describing the number of infectives is distinctive because it has two peaks. Finally, notice that all three isolation strategies result in the same value $S(T)=258$, as must be the case for all of them to be optimal.

Interestingly, this non-uniqueness means that policy makers have the freedom to optimize other cost functions in addition to (2). For example, as mentioned earlier, in this case it is possible to simultaneously minimize the total outbreak size, as well as the average number of infected individuals per unit time over the entire outbreak (which is a measure of its "peakedness"). The optimal strategy for doing so is to isolate individuals with maximal effort for as long as possible (Appendix).

The top panel of Fig. 2 also emphasizes the difference between the optimal isolation only policy and the optimal vaccination only policy. Notice that even though the dotted isolation strategy does not begin until the epidemic is well under way, it still minimizes the total number of infectives (and therefore also the total infectious burden). This result is very different from the vaccination results.

The bottom panel in Fig. 2 shows the results for three different isolation strategies for the mixed model. All three scenarios use an optimal vaccination strategy with $z_{\max }=2500$. All of the isolation strategies isolate 350 infectives, but not all of these strategies are optimal. The dashed and the solid curves illustrate dynamics for optimal control strategies and the total number of infected for both of these control strategies is 1861 . On the other hand, the control strategy denoted by the dotted line is not optimal (notice that the isolation control is off when the vaccination control is on) and consequently the total number of infected is larger, 1870 .

It is also worth highlighting the significance of how disease characteristics place upper bounds on the control variables. As an example, suppose that a disease of interest is such that the maximum possible rate of isolation is $u_{\max }=0.3$ as in Fig. 2. In this case (assuming $w_{\max }=1450$ as in Fig. 2), the optimal isolation policy will result in a total outbreak size of 4742 individuals. This yields the counterintuitive finding that, although we have enough resources to isolate 1450 individuals, and although there are only 10 infected individuals that initiate the outbreak, we are nevertheless unable to use isolation to prevent the epidemic. More surprising still, our resources turn out to be insufficient for the outbreak that develops. In other words, even though only 10 individuals start the epidemic, and even though we have enough resources to isolate 1450 individuals, our lack of ability to quickly isolate these initial 10 infections results in an outbreak so large that our resources are then insufficient. This clearly highlights the importance of constraints on the rate of isolation (and vaccination) in addition to constraints on the total amount of resources that are available. Indeed, given the 
Dynamics of Isolation Model for different Isolation Strategies,

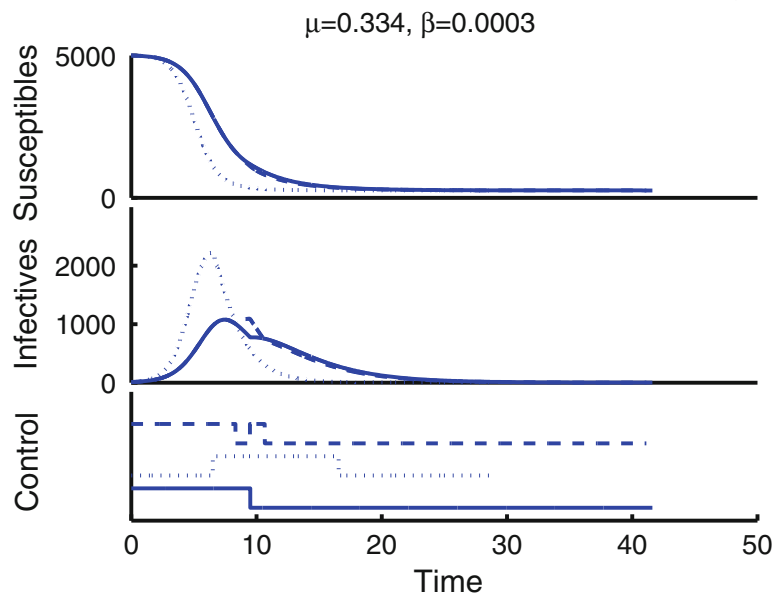

Dynamics of Mixed Model for different Isolation Strategies,

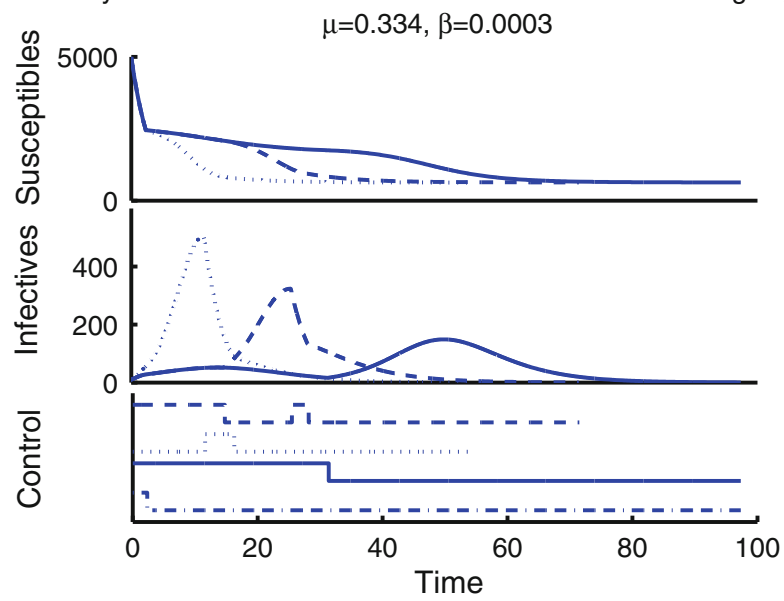

Fig. 2 Top panel: Dynamics of the isolation only model for three different, optimal isolation strategies. All three isolation strategies are purely bang-bang and switch between the values $u_{\min }=0$ and $u_{\max }=0.3$. The bottom plot shows the form of the different control strategies. The control strategies have been plotted offset from each other. The parameters used in these simulations are $I_{0}=10, S_{0}=5000, u_{\max }=0.3$, $\mu=0.334, w_{\max }=1450$ and $\beta=0.0003$. Bottom panel: Dynamics of the mixed model for three different isolation strategies. All three isolation strategies are purely bang-bang and switch between the values $u_{\min }=0$ and $u_{\max }=0.3$. All three isolation strategies isolate 350 infected. The vaccination strategy is the same for all three cases. The bottom plot shows the form of the different control strategies. The control strategies have been plotted offset from each other. The solid curve and the dashed curve denote optimal strategies, the dotted curve shows a non-optimal strategy. The dash-dot curve in the bottom plot is the vaccination strategy, $u_{v}$. The parameters used in these simulations are $I_{0}=10, S_{0}=5000, u_{\max }=0.3$, $\mu=0.334, w_{\max }=350, z_{\max }=2500$ and $\beta=0.0003$

likelihood of a large amount of uncertainty in $u_{\max }$, these considerations provide a justification for using up all of the isolation resources as quickly as possible, despite the fact that ultimately this might not reduce total outbreak size any more that using 
all the resources in some other temporal fashion, just in case $u_{\max }$ is larger than we think.

The above observations suggest a number of interesting areas that would benefit from further analysis. For example, the non-uniqueness of the optimal isolation policy, coupled with the fact that various optimal strategies yield dramatically different dynamics, suggests that aspects of the outbreak "shape" should be included in the optimization problem, and not simply outbreak size or infectious burden. It is also interesting to note that in the General Problem discussed in this paper, the values of $u_{\max }, z_{\max }$ and $w_{\max }$ are predetermined and fixed. It would be worthwhile to investigate the effects of varying these parameters and describing the interplay between $u_{\max }$ and the maximum possible number of isolated $\left(w_{\max }\right)$ and vaccinated $\left(z_{\max }\right)$ individuals. For example, if the optimal isolation policy is non-unique, then increasing the value of $u_{\max }$ (while keeping $w_{\max }$ fixed) will not change the value of the cost function provided that the new optimal control is still not unique. Conversely, increasing $u_{\max }$ (while keeping $z_{\max }$ fixed) will always improve the vaccination only policy. The effect of changing $u_{\max }$ for the mixed policy is not as easily understood nor is the effect of changing both $u_{\max }$ and $w_{\max }$ (or $z_{\max }$ ). Furthermore, it might also be interesting to examine what happens if the cost associated with using a control depends on the value of $w_{\max }$ (or $z_{\max }$ ) and not just on the total number of individuals that are isolated (or vaccinated). For instance, even if there is a fixed stockpile of vaccine, it is presumably more costly (in terms of needing nurses etc) to have a high vaccination rate than a low one at any given time. Lastly, the solution to the mixed model presented here assumes that the maximum allowed number of isolated and vaccinated individuals are constrained separately. It would be interesting to determine how to optimally divide a total resource pool into separate isolation and vaccination resource pools. This would be useful for example, when only the total amount of resources have been allocated and the policy-maker is free to choose how to divide these resources between vaccination and isolation.

Open Access This article is distributed under the terms of the Creative Commons Attribution Noncommercial License which permits any noncommercial use, distribution, and reproduction in any medium, provided the original author(s) and source are credited.

\section{Appendix}

The remaining details of the proofs of Theorems 1, 2 and 3 are presented here.

\section{Details for Proof of Theorem 1}

To derive Eq. 13 from Eq. 9 first note that Eq. 8 can be rearranged to give (i) $-\dot{S}=\beta S I$ and (ii) $I=-\frac{\dot{S}}{\beta S}$. Substituting these two expressions into Eq. 9 gives,

$$
\dot{I}=-\dot{S}+\frac{\mu}{\beta} \frac{\dot{S}}{S}-u_{i} I
$$


Rearranging Eq. 37 and integrating from $t=t_{0}$ to $t=T$ results in:

$$
\int_{t_{0}}^{T} u_{i} I \mathrm{~d} t=S_{0}-S(T)+I_{0}-I(T)+\frac{\mu}{\beta} \ln \left(\frac{S(T)}{S_{0}}\right) .
$$

The only detail that remains to be proved for Theorem 1 is that the optimal isolation strategy for the unconstrained problem is $u_{i}^{*} \equiv u_{\max }$.

Claim 1.1 The optimal control for Problem 1 with $w_{\max }=\infty$ is $u_{i}^{*} \equiv u_{\max }$.

Proof The PMP relations for Claim 1.1 are given by the PMP relations in Sect. 4 with $\lambda_{w}=0$. Substituting Eq. 12 into Eq. 11 gives,

$$
\begin{aligned}
\dot{\lambda}_{S} & =-\left(-\lambda_{0}-\lambda_{S}+\lambda_{I}\right) \beta I \\
& =\dot{\lambda}_{I} \frac{I}{S}-\lambda_{I}\left(\mu+u_{i}\right) \frac{I}{S}
\end{aligned}
$$

The first step is to show that the optimal control is purely bang-bang (that is, it has no singular components). Since, by Eq. $10, \lambda_{I}$ is a constant, if $u_{i}$ is singular then it must be singular on the entire interval $[0, T]$. This implies, by Eq. 39, that $\lambda_{S}$ is constant and since $\lambda_{S}(T)=0$ it must be that $\lambda_{S} \equiv 0$. Equation 11 then gives that $\lambda_{0}=0$. This is not possible since $\left(\lambda_{0}, \lambda_{I}(t), \lambda_{S}(t)\right)$ must be nonzero for all $t \in[0, T]$. Therefore, $u_{i}^{*}$ cannot be singular.

The optimal control will be determined once the sign of $\lambda_{I}$ is determined. To determine the sign of $\lambda_{I}$, use the transversality condition $\lambda_{S}(T)=0$. Since $\lambda_{I}$ is a constant, Eq. 12 gives that $\lambda_{I}=\frac{\left(\lambda_{0}+\lambda_{S}\right) \beta S}{\beta S-u-\mu}=\frac{\lambda_{0} \beta S(T)}{\beta S(T)-u_{i}(T)-\mu}$. This implies that $\operatorname{sign}\left(\lambda_{I}\right)=\operatorname{sign}\left(S(T)-\frac{u_{i}(T)+\mu}{\beta}\right)$. By Eq. $1, \lambda_{I}$ is negative if and only if $\dot{I}(T)<0$. Since $T$ is the smallest time that $I=0.5$ (and $I(0)>0.5$ ) it must be that $\dot{I}(T)$ is negative. Therefore, $u_{i}^{*} \equiv u_{\max }$.

\section{Details for Proof of Theorem 2}

The only detail that remains to be proved for Theorem 2 is that the vaccination strategy $u_{v} \equiv 0$ is not optimal.

Claim 2.1 The control $u_{v} \equiv 0$ is not a solution for Problem 2.

Proof From the PMP relations given in Sect. 5, if $u_{v}^{*} \equiv 0$ then $\lambda_{z} \leq \lambda_{S}$. Also, since $u_{v} \equiv 0$ means that none of the vaccination resources are used, it must be that $\lambda_{z}=0$. This implies that $\lambda_{S} \geq 0$.

Furthermore, by Eq. 14, $u_{v}^{*} \equiv 0$ implies that $\dot{\lambda}_{I} \equiv 0$. Rearranging Eq. 16 and setting $t=T$ gives $\lambda_{I}(\beta S(T)-\mu)=\lambda_{0} \beta S(T)$. There are two possible scenarios:

(i) If $\lambda_{0}=0$ then $\lambda_{I} \equiv 0$ but since $\lambda_{S}(T)=0$ this implies that the adjoint vector is zero at $t=T$. Therefore this scenario is not possible. 
(ii) If $\lambda_{0}=1$ then $\lambda_{I}<0$ (since $S(T)<\frac{\mu}{\beta}$ ) and this implies, by Eq. 14, that $\dot{\lambda}_{S}>0$. Therefore, since $\lambda_{S}(T)=0$, it must be $\lambda_{S}(t)<0$ for all $t<T$. But this contradicts the fact that $u_{v}^{*} \equiv 0$ implies that $\lambda_{S} \geq 0$.

\section{Details for Proof of Theorem 3}

We now prove the claims in Sect. 6 (i.e., Claims 3.1-3.6) that were used to prove Theorem 3. Throughout this section the notation specified in Remark 3 will hold and the PMP relations will be the ones given Sect. 6.

Claim 3.1 If $u_{v}$ is singular on some interval $J$ then $u_{i}$ is not singular on $J$ and $u_{i}=0$ on $J$.

Proof Suppose $u_{v}$ is singular on some interval $J$, then $\dot{\lambda}_{S}=0$ and therefore Eq. 27 implies that $\lambda_{I}=\lambda_{w} \frac{u_{i}}{\mu+u_{i}}$. Now if $\lambda_{w}=0$ then $\lambda_{I}=0$. Since $\dot{\lambda}_{S}=0$ it must be that $\lambda_{I}=\lambda_{0}+\lambda_{z}$ and therefore $\lambda_{0}=-\lambda_{z}$. Furthermore, since $T \in J$ (see Remark 5) it must be that $\lambda_{z}=0$ (because $\lambda_{S}(T)=0$ ) so the adjoint vector is identically zero on $J$. Since this is not possible it must be that $\lambda_{w}<0$ and therefore $\lambda_{w}<\lambda_{w} \frac{u_{i}}{\mu+u_{i}}=\lambda_{I}$ so $u_{i}=0$.

Remark 5 If $u_{v}$ is singular then $\dot{\lambda}_{S}=0$. The only way for $u_{v}$ to change from being singular to being nonsingular is if $\lambda_{I}$ becomes nonconstant. But, by Eq. 28 , if both $\dot{\lambda}_{S}=0$ and $\dot{\lambda}_{I}=0$ then $\dot{\lambda}_{I}$ must remain zero. This implies that if $u_{v}$ is singular on some maximal interval $J$ then $T \in J$.

Claim 3.2 If $u_{i}$ is singular on an interval $J$, then $u_{v}=0$ on $J$.

Proof If $u_{i}$ is singular on some interval $J$, then $\dot{\lambda}_{I}=0$ on $J$. This implies by Eq. 27 , that either $u_{v}$ is singular or $u_{v}=0$ on $J$. Since by Claim 7, $u_{i}$ and $u_{v}$ cannot be singular at the same time, it must be that $u_{v}=0$ on $J$.

Claim 3.3 When $u_{i}$ is singular, denote it by $u_{s}$. If $u_{i}$ has a singular component then $u_{i}$ has one of the following forms:

$$
\begin{aligned}
u_{\max } & \rightarrow u_{\mathrm{s}} \\
u_{\mathrm{s}} & \rightarrow u_{\mathrm{s}} .
\end{aligned}
$$

Proof Pick any $\tau \in J$. Claim 7 follows directly from the following two observations. Firstly, notice that if $u_{i}$ is singular on some interval $J$ then, by Eq. 27, $\dot{\lambda}_{S} \geq 0$ on $J$. This implies that $u_{v}(t)=0$ for all $t>\tau$ and therefore, by Eq. $27, \dot{\lambda}_{I}(t)=0$ for all $t>\tau$. That is if $u_{i}$ is singular on $J$ then $T \in J$. Secondly, by Eq. 27, $\dot{\lambda}_{I} \geq 0$ (even when $u_{i}$ is not singular).

Claim 3.4 If there exists a $t_{s} \geq 0$ such that $u_{i}$ is constant for all $t \in\left(t_{s}, T\right]$ then $u_{v}(t)=u_{v}^{*}(t)$ for all $t \in\left(t_{s}, T\right]$.

Proof Once $t>t_{S}$ the mixed isolation-vaccination model becomes a vaccination only model and therefore the optimal $u_{v}$ is the optimal control for the vaccination only model with parameters $\tilde{t}_{0}=t_{s}, \tilde{z}_{\max }=z_{\max }-\int_{t_{0}}^{t_{s}} u_{v} S_{\left[u_{i}, u_{v}\right]} \mathrm{d} t$ and $\tilde{\mu}=\mu+u_{i}(T)$. 
Claim 3.5 If there exists a $t_{s} \geq 0$ such that $u_{v}(t)=0$ for all $t \in\left(t_{s}, T\right]$ then $u_{i}(t)=u_{i}^{*}(t)$ for all $t \in\left(t_{s}, T\right]$.

Proof Once $t>t_{s}$ the mixed isolation-vaccination model becomes an isolation only model with parameters $\tilde{t}_{0}=t_{s}, \tilde{w}_{\max }=w_{\max }-\int_{t_{0}}^{t_{s}} u_{i} I_{\left[u_{i}, u_{v}\right]} \mathrm{d} t$ and $\tilde{\mu}=\mu$. Therefore the optimal $u_{i}$ is the optimal control for the isolation only model.

Claim 3.6 Let $J$ be any maximal interval such that

(i) $u_{i}=u_{\max }$ on $J$ and

(ii) $u_{i}$ is nonsingular on $J$.

If $T \notin J$ then $u_{v}=u_{\max }$ on $J$.

Proof Conditions i and ii imply that $\lambda_{I}(t)<\lambda_{w}$ for all $t \in J$. Therefore, by Eq. 27, $\dot{\lambda}_{S}(t)>0$ for all $t \in J$. Therefore, while $t \in J, u_{v}$ must have one of the following forms:

$$
\begin{aligned}
u_{\max } & \rightarrow u_{\max }, \\
u_{\max } & \rightarrow 0, \\
0 & \rightarrow 0 .
\end{aligned}
$$

Let $t_{1} \in J$. Suppose $u_{v}(t)=0$ for all $t \in \tilde{J}=\left\{t \mid t>t_{1}\right.$ and $\left.t \in J\right\}$ then, by Eq. 27, $\dot{\lambda}_{I}=0$ on $\tilde{J}$. This implies that $\max \left\{\lambda_{I}(t) \mid t \in \tilde{J}\right\}<\lambda_{w}$, since if $\max \left\{\lambda_{I}(t) \mid t \in\right.$ $\tilde{J}\} \geq \lambda_{w}$ either $\mathrm{i}$ or ii would be violated. But if $\max \left\{\lambda_{I}(t) \mid t \in \tilde{J}\right\}<\lambda_{w}$ then either $J$ is not maximal or $T \in J$. Since both of these possibilities lead to a contradiction, the only possible form for $u_{v}$ is $u_{v}=u_{\max }$ on $J$.

Isolation policy that minimizes average number of infectives

By introducing the variable $m=t$, the problem of minimizing the average number of infectives becomes the problem of finding the control $u_{i}$ that maximizes $\frac{S(T)-S_{0}}{m}$ subject to the constraints that $I(T) \leq 0.5$ and $w(T) \leq w_{\max }$. The PMP provides the following relations:

The Hamiltonian is

$$
H(t)=\beta S I\left(\lambda_{I}-\lambda_{S}\right)+\left(\lambda_{w}-\lambda_{I}\right) u_{i} I-\lambda_{I} \mu I+\lambda_{m}=-\dot{\lambda}_{I}+\lambda_{m}=0,
$$

where the adjoint variables are defined by, $\dot{\lambda}_{S}=-\beta I\left(\lambda_{I}-\lambda_{S}\right), \dot{\lambda}_{m}=0, \dot{\lambda}_{w}=0$ and $\dot{\lambda}_{I}=-\beta I\left(\lambda_{I}-\lambda_{S}\right)-\left(\lambda_{w}-\lambda_{I}\right)+\lambda_{I} \mu$. The transversality conditions are

$$
\left(\lambda_{0}, \lambda_{S}(T), \lambda_{I}(T), \lambda_{w}, \lambda_{m}\right)=\left(\lambda_{0}, \frac{\lambda_{0}}{m}, \lambda_{I}(T), \lambda_{w}, \lambda_{0} \frac{S_{0}-S(T)}{m^{2}}\right),
$$

where $\lambda_{I}(T), \lambda_{w} \leq 0$. The optimal control therefore satisfies

$$
u_{i}^{*}= \begin{cases}u_{\max } & \lambda_{w}-\lambda_{I}>0 \\ ? & \lambda_{w}-\lambda_{I}=0 \\ 0 & \lambda_{w}-\lambda_{I}<0\end{cases}
$$


The first step is to show that the optimal control is strictly bang-bang. Suppose $u_{i}^{*}$ is singular on an interval $J$, then $\lambda_{w}-\lambda_{I}=0$ on $J$. This implies that $\dot{\lambda}_{I}=0$ on $J$. By Eq. 40, this implies that $\lambda_{m}=0$ which (by the transversality conditions) implies that $\lambda_{0}=0$. Furthermore, since $H=0$ and $\dot{\lambda}_{m}=0$ this implies that $T \in J$. Substituting $\left.\left(\lambda_{0}, \lambda_{S}, \lambda_{I}, \lambda_{w}, \lambda_{m}\right)\right|_{t=T}=\left(0,0, \lambda_{w}, \lambda_{w}, 0\right)$ into $H=0$ gives that $\lambda_{w}=0$ and therefore the optimal control must be strictly bang-bang (since the adjoint vector must always be nonzero).

Since $\lambda_{m} \geq 0$, Eq. 40, implies that $\dot{\lambda}_{I} \geq 0$ and therefore the switch function is non-increasing. This implies that the optimal control is either $u_{i}^{*} \equiv u_{\max }, u_{i}^{*} \equiv 0$ or $u_{i}^{*}$ switches once from $u_{\max }$ to 0 . To further constrain the form of the optimal control, consider the case when $\lambda_{w}=0$. Since $\lambda_{I}(T) \leq 0$ and $\dot{\lambda}_{I}>0$ it must be that $\lambda_{I}(t)<0$ for all $t<T$ and therefore $\mathrm{SF}(t) \geq 0$. Therefore, if $\lambda_{z}=0$ the optimal control is to isolate with maximal effort for the entire epidemic.

Now consider the case when $\lambda_{w}<0$. If $\lambda_{w}<0$ then all of the control resources are used therefore the optimal control is to isolate with maximal effort until all of the resources are used.

Combining both cases, gives that the optimal control is to isolate with maximal effort until either all of the resources are used or the epidemic is over.

Simulations for more detailed models

Here we consider two more detailed versions of model (1). The first version includes more detailed isolation dynamics and allows for the possibility that isolated individuals may still transmit infection. The more detailed isolation model is described by the following set of equations:

$$
\begin{aligned}
\dot{S} & =-\left(\beta I+\beta_{J} J\right) S-u_{v} S, \\
\dot{I} & =\left(\beta I+\beta_{J} J\right) S-\left(\mu+u_{i}\right) I, \\
\dot{J} & =u_{i} I-\mu J
\end{aligned}
$$

where $S, I$ and $J$ are the numbers of susceptible, infected and isolated hosts, $\beta$ is the transmission constant for infected hosts, $\beta_{J}$ is the transmission constant for isolated hosts, $\mu$ is the per capita loss rate of infected individuals through both mortality and recovery, and $u_{i}$ is the per capita rate of isolation.

Perhaps the most important result for the isolation model is that if there are not enough resources to isolate for the entire outbreak then any isolation strategy that uses the maximum amount of resources is optimal. Figure 3 suggests that this result also holds for the more detailed isolation model. More specifically, if $E_{i}^{*}$ is the number of individuals isolated when $u_{i} \equiv u_{\max }$, then Fig. 3 suggests that all isolation strategies that isolate $E_{i}<E_{i}^{*}$ result in the same outbreak size. It is important to emphasive that these results are only suggestive and that much more detailed analysis is required in order to make a stronger statement.

The second version includes more detailed vaccination dynamics and allows for the possibility that vaccinated individuals may still contract infection. The more detailed 


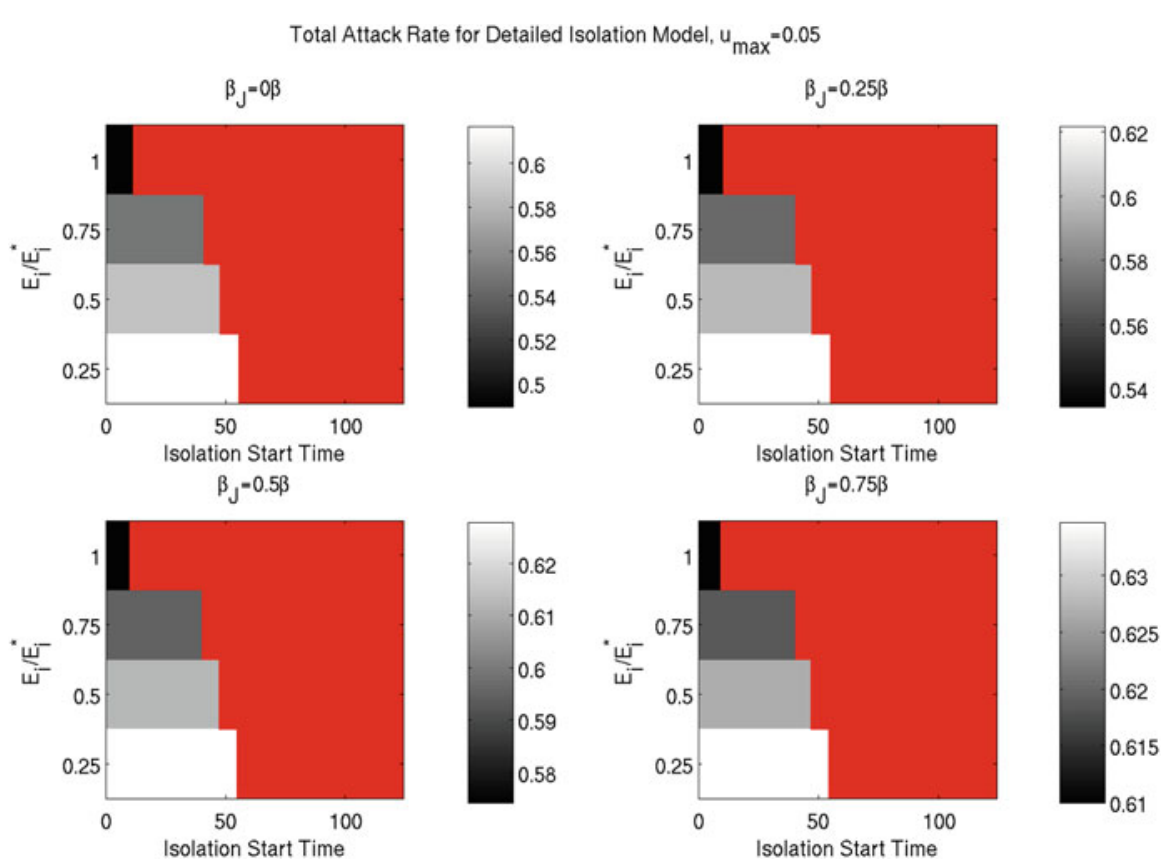

Fig. 3 Results suggesting that if there are not enough resources to isolate for the entire outbreak then any isolation strategy that uses the maximum amount of resources is optimal. Each panel corresponds to a different value of $\beta_{J}$. The upper left panel corresponds to $\beta_{J}=0$ and therefore corresponds to the situation when isolation is completely effective. The bottom right panel corresponds to $\beta_{J}=0.75 \beta$ and therefore corresponds to the situation when isolation is not very effective. $E_{i}^{*}$ denotes the total number of isolated individuals when isolation starts immediately and continues for the entire outbreak with maximal effort. Each panel shows the attack rate as a function of total isolated (normalized by $E_{i}^{*}$ ) and isolation start time. Each point on the panels was generated by beginning isolation at the time indicated on the horizontal axis and continuing to isolate with maximal effort until the number isolated is the value indicated on the vertical axis. Once the total isolated has reached the number indicated on the vertical axis, no more hosts are isolated. If the outbreak ends before enough individuals have been isolated, the corresponding point on the plot has the same shade of gray as the background. Thus the background gray areas on the panels should be ignored. All four panels suggest that all isolation strategies that isolate $E_{i}<E_{i}^{*}$ result in the same outbreak size (i.e., the shade of gray is constant for all isolation start times that correspond to the same value of $E_{i}$ ). Parameters used are $S_{0}=100000, I_{0}=10, \mu=\frac{1}{3.3}, \beta=\frac{1.6 \mu}{S_{c} a 0}, \beta_{v}=0.8 \beta, u_{\max }=0.05$

vaccination model is described by the following set of equations:

$$
\begin{aligned}
\dot{S} & =-\left(\beta I+\beta_{v} I_{v}\right) S-u_{v} S, \\
\dot{S}_{v} & =u_{v} S-(1-r)\left(\beta I+\beta_{v} I_{v}\right) S_{v}, \\
\dot{I} & =\left(\beta I+\beta_{v} I_{v}\right) S-\left(\mu+u_{i}\right) I, \\
\dot{I}_{v} & =(1-r)\left(\beta I+\beta_{v} I_{v}\right) S_{v}-\left(\mu+u_{i}\right) I_{v},
\end{aligned}
$$

where $S$ and $I$ are the numbers of susceptible and infected hosts, $S_{v}$ and $I_{v}$ are the numbers of vaccinated susceptible and vaccinated infected hosts, $r$ represents the reduction in susceptibility of vaccinated individuals, $\beta$ is the transmission constant for infected 
Total Attack Rate for Detailed Vaccination Model, $u_{\max }=0.05$
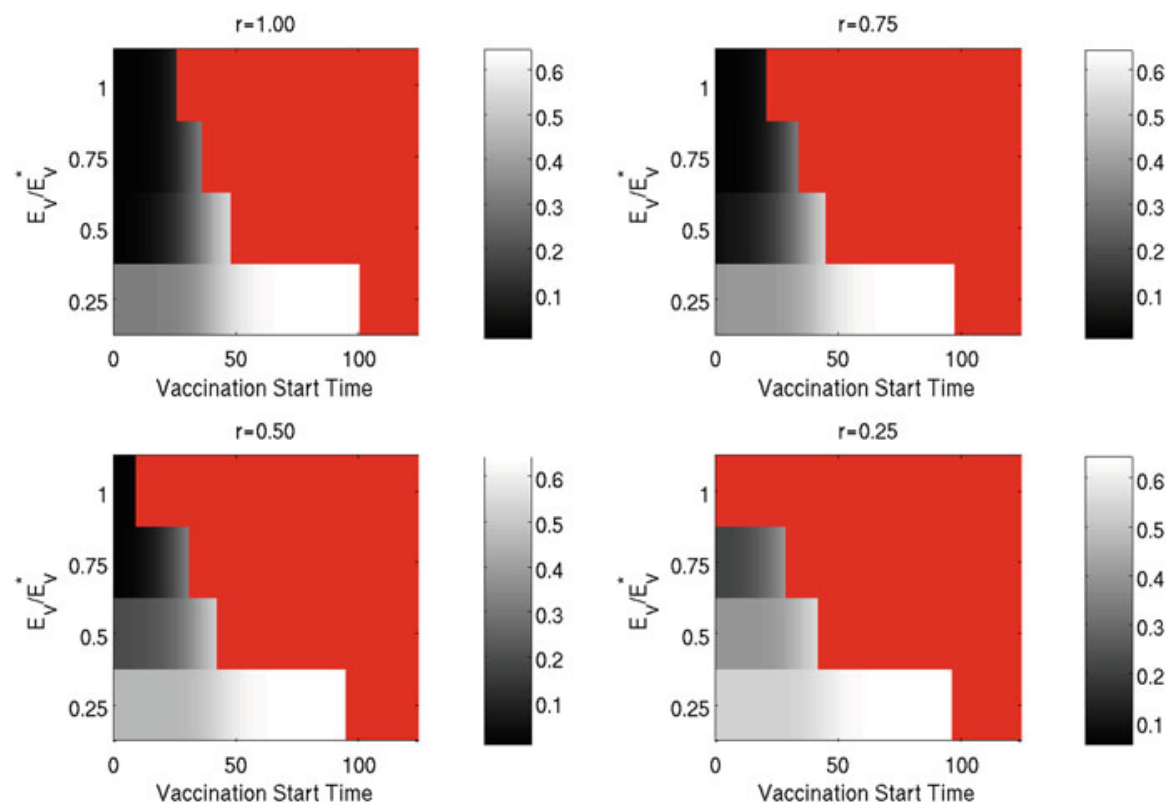

Fig. 4 Results suggesting that it is optimal to start vaccinating immediately and to vaccinate for the entire outbreak. Each panel corresponds to a different value of $r$. The upper left panel corresponds to $r=1$ and therefore corresponds to the situation when vaccination is completely effective. The bottom right panel corresponds to $r=0.25$ and therefore corresponds to the situation when vaccination is not very effective. $E_{v}^{*}$ denotes the total number of vaccinated individuals when vaccination starts immediately and continues for the entire outbreak with maximal effort. Each panel shows the attack rate as a function of total vaccinated (normalized by $E_{v}^{*}$ ) and vaccination start time. Each point on the panel was generated by beginning vaccination at the time indicated on the horizontal axis and continuing to vaccinate with maximal effort until the number vaccinated is the value indicated on the vertical axis. Once the total vaccinated has reached the number indicated on the vertical axis, vaccination is stopped. If the outbreak ends before enough individuals have been vaccinated, the point is the same shade of gray as the background. Thus the background gray areas on the panels should be ignored. All four panels suggest that starting vaccination immediately is best (the smaller the vaccination start time the smaller the attack rate, or in other words the shade of gray lightens as we move from left to right). All four panels show that, of the vaccination strategies represented on the panels, vaccinating for the entire outbreak results in the lowest attack rate. For the bottom right panel, of the vaccination strategies considered the only one that vaccinates $E_{v}^{*}$ individuals is the one that the vaccinates for the entire outbreak. Therefore, the entire upper row (except the point corresponding to a vaccination start time of $t=0)$ is red. Parameters used are $S_{0}=100000, I_{0}=10, \mu=\frac{1}{3.3}, \beta=\frac{1.6 \mu}{S_{0}}, \beta_{v}=0.8 \beta$, $u_{\max }=0.05$

hosts, $\beta_{v}$ is the transmission constant for vaccinated infected hosts, $\mu$ is the per capita loss rate of infected individuals through both mortality and recovery, and $u_{v}$ is the per capita rate of vaccination. Perhaps the most important result for the vaccination model is that it is optimal to begin vaccination immediately and to vaccinate for the entire epidemic. Figure 4 suggests that this result also holds for the more detailed vaccination model. More specifically, if $E_{v}^{*}$ is the number of individuals vaccinated when $u_{v} \equiv u_{\max }$, then Fig. 4 shows that vaccination strategies that vaccinate the same 
number of individuals result in different outbreak sizes. Furthermore, of the strategies considered, it is always best to start vaccinating immediately and to continue vaccinating for longer. It is important to emphasize that these results are only suggestive and that much more detailed analysis is required in order to make a stronger statement.

\section{References}

Abakuks A (1972) Optimal policies for epidemics. D.Phil. Thesis, University of Sussex

Abakuks A (1973) An optimal isolation policy for an epidemic. J Appl Probab 10:247-262

Abakuks A (1974) Optimal immunization policies for epidemics. Adv Appl Probab 6:494-511

Agrachev AA, Sachkov YL (2004) Control theory from the geometric viewpoint. In: Encyclopedia of mathematical sciences, vol 87. Springer-Verlag, New York

Anderson RM, May RM (1991) Infectious diseases of humans: dynamics and control. Oxford Science Publications, Oxford

Behncke H (2000) Optimal control of deterministic epidemics. Optim Control Appl Methods 21:269-285

Berkovitz LD (1974) Optimal control theory. In: Applied mathematical sciences, vol 12. Springer-Verlag, New York

Greenhalgh D (1988) Some results on optimal control applied to epidemics. Math Biosci 88:125-158

Kamien MI, Schwartz NL (2003) Dynamic optimization. In: The calculus of variations and optimal control in economics and management, 2nd edn. Advanced textbooks in economics, vol 31. Elsevier, North Holland

Lipsitch M, Cohen T, Murray M, Levin BR (2007) Antiviral resistance and the control of pandemic influenza. PLoS Med 4(1):e15. doi:10.1371/journal.pmed.0040015

Morton R, Wickwire KH (1974) On the optimal control of a deterministic epidemic. Adv Appl Probab 6:622-635

Oostvoigel PM et al (1994) Poliomyelitis outbreak in an unvaccinated community in the Netherlands, 1992-93. Lancet 344:665-670

Pontryagin LS et al (1964) The mathematical theory of optimal processes. International series of monographs in pure and applied mathematics, vol 55. Macmillan, New York

Wickwire KH (1975) Optimal isolation policies for deterministic and stochastic epidemics. Math Biosci 26:325-346

Wickwire KH (1977) Mathematical models for the control of pests and infectious diseases: a survey. Theor Popul Biol 11:182-238 